\title{
Drug-resistant viruses may repair impaired fitness by mutations outside the drug target site
}

\section{Lifeng Cai \& Shibo Jiang ${ }^{\dagger}$}

†Author for correspondence: Lindsley F Kimball Research Institute, New York Blood Center, New York, NY 10065, USA m Tel. : +1 2125703058 m Fax: +1 2125703099 m sjiang@nybloodcenter.org

Evaluation of: Ray N, Blackburn LA, Doms RW: HR-2 mutations in human immunodeficiency virus type 1 gp4l restore fusion kinetics delayed by HR-1 mutations that cause clinical resistance to enfuvirtide. J. Virol. 83(7), 2989-2995 (2009). During the treatment of HIV infection with viral fusion inhibitors derived from HIV-1 gp41 heptad repeat (HR)-2 regions, drug-induced mutations have been observed not only in the HR-1 region that contains the target sites for the inhibitors, but also the HR-2 region, which is outside the inhibitor target site. Using a kinetic cell-cell fusion assay, Ray et al. have demonstrated that the HR-1 mutation, which helps viruses to escape from treatment challenge, significantly delays fusion kinetics, leaving viruses more vulnerable to the host immune defense system, thus reducing the fitness of the virus. However, the mutations in the HR-2 region restore the delayed fusion kinetics close to its original level, partially repairing the impaired fitness caused by the mutations in the drug target site. The work sheds new light on the mechanism of HIV-1 drug resistance, which may be used for the development of new HIV fusion inhibitors with improved efficacy and drug resistance profiles.

In this article we will review the recent publication by Ray, Blackburn and Doms [1]. Mutation is a common strategy used by microbes to escape from treatment challenge, and the most distinguished among them is HIV-1. The super-plasticity of its genome enables HIV-1 to develop resistance against almost all current antiretroviral therapies. Unlike other medications that target a distinct motif, the peptidic HIV-1 fusion inhibitors, derived from the HIV-1 gp41 heptad repeat (HR)-2 region, such as C34 and T20 (enfuvirtide), target multiple sites in the HR-1 domain and even beyond it [2]. They can induce multiple resistance mutations in HR-1 or other regions $[3,4]$. It is easy to interpret the resistance mechanism of viruses with mutations in the gp 41 HR-1 as this region contains the drug target sites. However it is relatively difficult to explain the role of resistance-associated mutations located outside the drug target site since these mutations may have no direct effect on binding of the inhibitors to their targets. For example, some mutations in T20-resistant viruses are located in the HR-2 region [1,4]. Strikingly, Baldwin et al. have demonstrated that one HIV-1 HR-1-HR-2 double mutant is not only highly resistant to T20 but also dependent on the T20 peptide for its replication [5].

Ray et al. identified a panel of T20-resistant HIV-1 strains from five patients who had failed to respond to treatment with T20. All the resistant virus strains had mutations in the HR-1 region that contains the T20 binding site and resistance could be attributed to the mutation-caused reduction of binding affinity between T20 and the HR-1 region. They also identified a number of mutations in the HR-2 region. Since the mutations in the HR-2 are outside the drug target site, the mechanism could not be explained as a disruption of drug-target interaction because these mutations should have no direct effect on drug-target binding affinity. In order to understand the mechanism of resistance due to these mutations, the authors used a kinetic cell-cell fusion assay to quantitatively measure the fusion process in situ. They generated effector cells expressing the HIV-1 envelope glycoprotein (Env) bearing HR-1 mutations with or without HR-2 mutations and compared their fusion kinetics with target cells that express CD4 and co-receptors. The authors demonstrated that the introduction of a resistance mutation in the HR-1 region alone, which reduced the interaction between T20 and HR-1 and helped the viruses escape from treatment challenge, prolonged the fusion processes by up to 19 min when compared with those without such mutations. When the HR-2 mutations were introduced into the same Env with HR-1 mutations, the delayed fusion kinetics was restored to the original level.

These authors have previously demonstrated that the introduction of T20-resistant mutations into the gp41 HR-1 region of HIV-1 LAI and
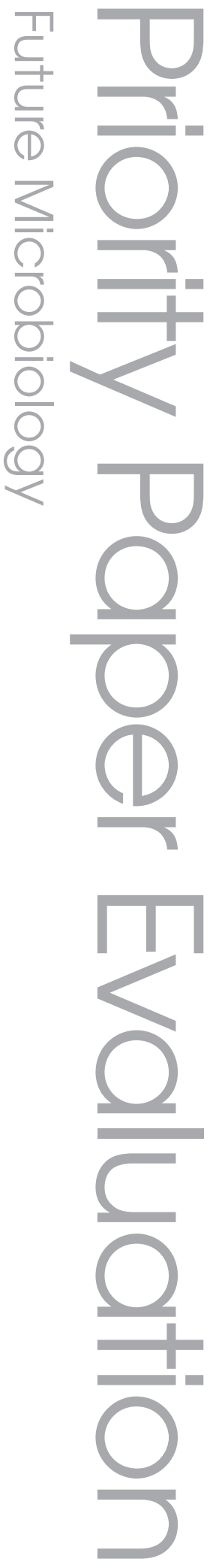

fulure medicine $_{\text {fisg }}^{\text {pis }}$ 
YU-2 strains results in a prolonged fusion process and increases sensitivity to neutralizing monoclonal antibodies targeting gp41 [6], presumably because the prolonged fusion process may over expose the HIV-1 gp41 prefusion state and make the virus be more vulnerable to the host immune defense system, thus resulting in a reduced fitness of the virus. In the present study, the authors generated a panel of pseudotype viruses bearing HR-1 mutations with or without HR-2 mutations in the Env of viruses from the same patient or another patient (i.e., a homologous or heterologous Env background) and tested their susceptibility to neutralizing monoclonal antibodies targeting adjuvant gp 41. They found that the viruses exhibited increased sensitivity to neutralizing antibodies if the HR-1 and HR-2 mutations were introduced into gp41 with a heterologous Env background, consistent with their previous observation [6]. However, no significant difference in antibody susceptibility was observed when the HR-1 and HR-2 mutations were introduced into gp41 with a homologous Env background. This suggests that in vivo evolution of viral resistance and fitness are genetic context dependent. When viruses evolve to adapt resistance, the mutations are selected to balance between drug resistance and virus fitness in a given viral genome context.

\section{Conclusion \& future perspective}

Studying the roles of mutations inside and outside the drug target site provides additional information to understand the mechanism of drug action, which can be used for the development of new generation drugs. The peptidic HIV fusion inhibitors, such as T20 and C34, target multiple sites in the HIV-1 gp41 HR-1 domain and may be even beyond it, in contrast to most other antiHIV drugs, which have a target located in a distinct motif [2,7]. HIV-1 Env-mediated membrane fusion is a kinetically limited process and mutations associated with T20 resistance can delay the fusion kinetics, thus enhancing sensitivity to neutralizing antibodies targeting gp41 [6]. Using a kinetic cell-cell fusion assay, Ray et al. have demonstrated that the mutations in the HIV-1 gp41 HR-2 domain, which is outside the drug target site, serve to restore the delayed fusion kinetics caused by resistance mutations in the drug target, thus repairing the impaired fitness of viruses in the adapted environment. The work shed new light on the mechanism of viral resistance to HIV fusion inhibitors.

The mechanism elucidated here may be used for designing novel HIV-1 fusion inhibitors against T20-resistant strains. Recently, Izumi et al. have demonstrated that the same S138A mutation in the HR-2 region, which acts to restore the delayed viral fusion kinetics caused by the HR-1 mutations observed in this paper, could be introduced into T20 to fight against T20 resistant HIV-1 viruses [8]. Most T20-resistant HIV-1 variants carry mutations in residues 33-45 of the HR-1 domain, the primary binding site for T20 [9]. However, this region is not the primary binding site for many of the next generation HIV fusion inhibitors, such as sifuvirtide, which contains a pocket-binding domain that can interact with the hydrophobic pocket in the HR-1 domain. We found that sifuvirtide is more effective than T20 against T20-resistant strains [10]. We also found that a combination of sifuvirtide with T20 resulted in potent synergism impacting on inhibition of infection by T20-sensitive and resistant HIV-1 strains [11]. We have recently designed a peptide that contains the pocket-binding domain and lacks the sequence that binds to residues 33-45 of the HR-1 region and demonstrated that this peptide exhibits exceptional potency against T20-resistant variants [12]. All of these findings suggest that the study of the mechanism of resistance to HIV fusion inhibitors is very useful for the development of novel anti-HIV drugs with improved potency and resistance profile.

\section{Executive summary}

\section{Objectives of this study}

- To explore the mechanistic basis for T20 resistance and the role of mutations in the HIV-1 gp41 heptad repeat (HR)-2 region that is outside the drug target site.

\section{Methods}

- A cell-cell fusion assay using QT6 cells that express HIV-1 Env and $\beta$-lactamase as effector cells and Hela/CD4/CCR5 (JC53) cells loaded with the fluorescent dye CCF2-AM as target cells to monitor Env-mediated cell fusion kinetics.

- A temperature-arrested state assay to dissect the steps of Env-mediated cell fusion that are delayed in HR-1 mutants.

- A luciferase-based virus infection assay using pseudoviruses bearing various Env mutations to test their sensitivity to T20 and various neutralizing monoclonal antibodies.

\section{Conclusion}

- Mutations in HIV-1 gp41 HR-2, which is outside the drug target site, serve to restore the delayed fusion kinetics caused by resistance mutations in the drug target site, thus repairing the impaired fitness of viruses adapted to their environment. 


\section{Financial \& competing interests disclosure}

The authors have no relevant affiliations or financial involvement with any organization or entity with a financial interest in or financial conflict with the subject matter or materials discussed in the manuscript.
This includes employment, consultancies, honoraria, stock ownership or options, expert testimony, grants or patents received or pending, or royalties.

No writing assistance was utilized in the production of this manuscript.

\section{Bibliography}

1. Ray N, Blackburn LA, Doms RW: HR-2 mutations in human immunodeficiency virus type $1 \mathrm{gp} 41$ restore fusion kinetics delayed by HR-1 mutations that cause clinical resistance to enfuvirtide. J. Virol. 83(7), 2989-2995 (2009).

2. Liu S, Lu H, Xu Y et al.: Different from the HIV fusion inhibitor C34, the anti-HIV drug Fuzeon (T-20) inhibits HIV-1 entry by targeting multiple sites in gp 41 and gp120. J. Biol. Chem. 280 (12), 11259-11273 (2005).

3. Kilby JM, Hopkins S, Venetta TM et al: Potent suppression of HIV-1 replication in humans by T-20, a peptide inhibitor of gp41-mediated virus entry. Nature Med. 4(11), 1302-1307 (1998).

4. Xu L, Pozniak A, Wildfire A et al: Emergence and evolution of enfuvirtide resistance following long-term therapy involves heptad repeat 2 mutations within gp41. Antimicrob. Agents Chemother. 49(3), 1113-1119 (2005).

5. Baldwin CE, Sanders RW, Deng Y et al.: Emergence of a drug-dependent human immunodeficiency virus type 1 variant during therapy with the T20 fusion inhibitor. J. Virol. 78(22), 12428-12437 (2004).

6. Reeves JD, Lee FH, Miamidian JL et al.: Enfuvirtide resistance mutations: impact on human immunodeficiency virus envelope function, entry inhibitor sensitivity, and virus neutralization. J. Virol. 79(8), 4991-4999 (2005).

7. Liu S, Jing W, Cheung B et al.: HIV gp41 C-terminal heptad repeat contains multifunctional domains: relation to mechanisms of action of anti-HIV peptides. J. Biol. Chem. 282(13), 9612-9620 (2007).

8. Izumi K, Kodama E, Shimura K et al.: Design of peptide-based inhibitors for human immunodeficiency virus type 1 strains resistant to T-20. J. Biol. Chem. 284(8), 4914-4920 (2009).

9. Mink M, Mosier SM, Janumpalli S et al.: Impact of human immunodeficiency virus type $1 \mathrm{gp} 41$ amino acid substitutions selected during enfuvirtide treatment on gp 41 binding and antiviral potency of enfuvirtide in vitro. J. Virol. 79(19), 12447-12454 (2005).
10. He Y, Xiao Y, Song H et al:: Design and evaluation of sifuvirtide, a novel HIV-1 fusion inhibitor. J. Biol. Chem. 283(17), 11126-11134 (2008).

11. Pan C, Lu H, Qi Z et al:: Synergistic efficacy of combination of enfuvirtide and sifuvirtide, the first- and next-generation HIV-fusion inhibitors. AIDS 23(5), 639-641 (2009).

12. He Y, Cheng J, Lu H et al:: Potent HIV fusion inhibitors against enfuvirtide-resistant HIV-1 strains. Proc. Natl Acad. Sci. USA 105(42), 16332-16337 (2008).

\section{Affiliations}

- Lifeng Cai

Lindsley F Kimball Research Institute, New York Blood Center, New York, NY 10065, USA Tel.: +1 212570 3188;

Fax: +1 2125703099 ;

lcai@nybloodcenter.org

- Shibo Jiang Lindsley F Kimball Research Institute, New York Blood Center, New York, NY 10065, USA

Tel.: +1 212570 3058;

Fax: +1 212570 3099; sjiang@nybloodcenter.org 